\title{
Sequence Design Technique for Accurate Timing and Cell ID Estimation in Underwater Acoustic Cellular Systems with a High Doppler
}

\author{
Yeong Jun Kim ${ }^{1}$, Muhammad Asim ${ }^{2}$ (D) Tae Ho Im ${ }^{3}$ (D) and Yong Soo Cho ${ }^{2, *(\mathbb{D})}$ \\ 1 LG Electronics, Seoul 137-893, Korea; yjkim81@gmail.com \\ 2 Department of Electrical and Electronics Engineering, Chung-Ang University, Seoul 156-756, Korea; \\ muhammad3428@gmail.com \\ 3 Department of Information and Communication Engineering, Hoseo University, Asan-si 336-795, Korea; \\ taehoim@hoseo.edu \\ * Correspondence: yscho@cau.ac.kr; Tel.: +82-2-820-5299
}

check for

updates

Citation: Kim, Y.J.; Asim, M.; Im, T.H.; Cho, Y.S. Sequence Design Technique for Accurate Timing and Cell ID Estimation in Underwater Acoustic Cellular Systems with a High Doppler. Electronics 2021, 10, 2413. https://doi.org/10.3390/ electronics10192413

Academic Editors: Giovanni Andrea Casula and Alberto Gatto

Received: 30 July 2021

Accepted: 27 September 2021

Published: 2 October 2021

Publisher's Note: MDPI stays neutral with regard to jurisdictional claims in published maps and institutional affiliations.

Copyright: (c) 2021 by the authors. Licensee MDPI, Basel, Switzerland. This article is an open access article distributed under the terms and conditions of the Creative Commons Attribution (CC BY) license (https:/ / creativecommons.org/licenses/by/ $4.0 /)$.

\begin{abstract}
In underwater acoustic cellular (UWAC) systems, underwater equipment or sensor nodes (UE/SN) should perform downlink synchronisation and a cell search during the initial access stage using the preambles received from adjacent underwater base stations (UWBSs). The UE/SN needs to estimate accurate timing and cell ID (CID) using the received preambles, and synchronise with a serving UWBS, even in high-Doppler environments. In this paper, a sequence design technique for joint estimation of accurate timing and CID in UWAC systems with a high Doppler is proposed to decrease the receiver complexity and processing time. A generalised Zadoff-Chu sequence is proposed for the preamble design. This sequence is decomposed into multiple short sub-sequences to reduce the effect of Doppler shift on the timing and CID estimation. The performance loss caused by the short sequence length is compensated by combining the sub-sequences using the repetition property of the ZC sequence. The properties (autocorrelation and cross-correlation) of the proposed sequence are derived analytically in the presence of Doppler shift and compared with the simulation results. The simulation results reveal that the proposed technique performs better than existing techniques in both additive white Gaussian noise and multipath channels with a high-Doppler. It is concluded that the proposed technique is suitable for accurate timing estimation and CID detection in UWAC systems with a high Doppler.
\end{abstract}

Keywords: underwater acoustic cellular system; cell search; timing; Doppler

\section{Introduction}

During the last decade, underwater acoustic communication and networking techniques have been developed for subsea exploration, resource extraction, and defence missions [1-7]. Scientific advances have been made in multiple disciplines, such as surveillance, disaster prevention, oil and gas exploration, and environmental monitoring. Autonomous underwater vehicles (AUVs) or remotely operated vehicles, such as remote environment monitoring units, spray gliders, and slocum gliders, can be used to collect important data, such as real-time video, environmental data, and security data, over a long period of time [8,9]. To setup a connection among nodes in UWAC system, several communication media, such as cables, electromagnetic waves, optical waves, and acoustic waves have been used [1].

Acoustic waves are extensively used in UWAC systems, because they propagate well in underwater and can cover large distances. Acoustic waves tend to propagate through multipath trajectories owing to the reflections on the surface of the ocean and the refractions caused by the depth-varying sound speed. The temporal variability of the ocean as well as the low sound speed in water may induce significant Doppler shifts $[10,11]$. 
Consequently, the channel is affected by time and/or frequency dispersion. The attenuation of acoustic signals also increases with the frequency and range. The available bandwidth of an underwater channel is limited to the low-frequency region and depends on the transmission range and the frequency $[12,13]$. The capacity of a UWAC system based on OFDM was demonstrated to be better than that of a single-carrier system. A code-division multiple access (CDMA)-based UWAC system and a cellular underwater wireless optical CDMA system were proposed in [14-16].

Accurate position estimation in a UWAC system is quite challenging task because the performance of position estimation is dependent upon several factors such as multipath propagation, propagation delay, and Doppler shift. The global positioning system (GPS) cannot be applied in underwater environments because electromagnetic waves are severely attenuated in a UWAC system $[17,18]$. Position estimation techniques can be broadly divided into two categories: range-based techniques and range free techniques. The range-based techniques estimates the position using Angle of Arrival (AOA), Time of Arrival (TOA), Time Difference of Arrival (TDoA), and Received Signal Strength (RSS) [19-22]. Whereas the range free techniques includes 2D Area Localization Scheme (2D-ALS) and 3D-Multi-Stage Area Localization Scheme (3D-MALS) [23,24]. Commercially available UWAC position estimation techniques are Long Baseline (LBL) and Short Baseline (SBL) [25-30]. Recently, position estimation techniques based on machine learning were proposed in [31-38]. Table 1 summarizes the position estimation techniques.

Table 1. Position estimation techniques.

\begin{tabular}{cc}
\hline Name of Position Estimation Techniques & Algorithms \\
\hline Range based techniques & {$[19-22]$} \\
Range free based techniques & {$[23,24]$} \\
Commercial position estimation techniques & {$[25-30]$} \\
Machine learning based techniques & {$[31-38]$} \\
\hline
\end{tabular}

Similarly as in terrestrial cellular systems, an initial access procedure is required for an underwater equipment or sensor node (UE/SN) in UWAC systems to establish a communication link with an underwater base station (UWBS). To establish a communication link, the UE should perform downlink synchronisation and cell search by receiving synchronisation and broadcasting signals. Known sequences, termed as preamble, are generally transmitted from UWBSs to acquire synchronisation parameters and to detect the cell ID (CID) at the UE. Sequences can be broadly divided into two categories: binary and non-binary. The m-sequence, Hadamard sequence, and Gold sequence are well-known binary sequences. The m-sequence is known to have a good autocorrelation function. However, its cross-correlation function is not good. The cross-correlation function is considered good if the periodic cross-correlation values are small for all possible shifts between the pairs of sequences in the given set. The Hadamard sequence has an ideal cross-correlation function. However, its autocorrelation function is not good. The Gold sequence has a good cross-correlation function. However, it is sensitive to Doppler shift because, in the presence of Doppler shift, the peak value in the correlation function approaches zero at the correct timing or produces a large time shift from its correct position. Non-binary sequences such as the Zadoff-Chu (ZC) sequence have an ideal autocorrelation function and a good cross-correlation function. They can also support a large number of CIDs. However, they are sensitive to Doppler shift. A linear frequency modulated (LFM) waveform may be considered for a preamble design because its matched filter output produces a high peak in the presence of a large Doppler shift. The LFM waveform is widely used for surveillance applications, such as RADAR and SONAR, because the target is more likely to be detected in high-Doppler environments. However, in the case of a Doppler mismatch, its peak does not occur at the correct timing and is shifted by an amount that is proportional to the Doppler shift. The LFM waveform is known as the most Doppler-insensitive waveform because it can produce a significant output peak for a broad range of Doppler shifts. The 
LFM waveform can be used in the preamble design for UWAC systems if only preamble detection is required in the presence of a large Doppler shift $[39,40]$. However, the LFM waveform is not adequate for timing estimation in high-Doppler environments because it cannot produce a peak at the correct timing.

Synchronisation and cell search techniques mainly developed for Long-Term Evolutionbased systems cannot be directly applied to underwater channels because of the large discrepancy in propagation speed and bandwidth [41,42]. To successfully demodulate the data transmitted from a UWBS at the UE, we need the following synchronisation and cell search procedures: signal detection, physical cell ID (CID) detection, coarse/fine timing estimation, integer/fractional carrier frequency offset (CFO) estimation/compensation, and Doppler scale estimation/compensation. Because many synchronisation techniques for UWAC systems are already available [43-46], we focus on CID and timing estimation in this paper.

In [47], two different types of cell search techniques (CSTs), namely, linear frequency modulation with full bandwidth in the time domain (LFM-FT-CST) and linear frequency modulation in the frequency domain (LFM-FF-CST), were proposed to detect the CID and timing in UWAC systems. In both techniques, multiple LFM waveforms are generated by changing the frequency-sweeping parameters of the LFM waveform. In the LFM-FT-CST, the preambles are directly generated in the time domain with two different frequencysweeping parameters, one for each of the two halves of the preamble duration. In the LFM-FF-CST, the LFM waveforms are generated in the frequency domain with different frequency-sweeping parameters and converted into the time domain via an inverse discrete Fourier transform. Although these techniques can be used to detect the CID and timing in UWAC systems, the accuracy of timing estimation in high-Doppler environments is not high enough for fine timing. The timing estimate obtained by these techniques can be used for coarse timing, requiring an additional block for fine timing. The additional block for the implementation of fine timing increases the receiver complexity and processing time.

In this paper, a preamble design technique for joint estimation of accurate timing and CID in OFDM-based UWAC systems with a high Doppler is proposed to decrease the receiver complexity and processing time. Because the $Z C$ sequence itself is not adequate for high-Doppler environments, a generalised ZC sequence (G-ZCS) is proposed and decomposed into multiple short sub-sequences to reduce the Doppler effect on timing and CID estimation. The performance loss caused by the short sequence length is compensated by combining the sub-sequences using the repetition property of the $\mathrm{ZC}$ sequence. Multiple UWBSs in a UWAC system are distinguished by assigning different root indices to the G-ZCS. The autocorrelation and cross-correlation properties of the G-ZCS are analysed in the presence of Doppler shift and used to determine the number of possible root indices (CIDs) and cross-correlation (intercell interference) levels. In addition, the relationship between the G-ZCS and the LFM waveform is described. Finally, the performances of the previous and proposed techniques are evaluated using a simple three-cell model and a Bellhop channel simulator, which is widely used to simulate a multipath channel in underwater acoustic communication. The results are presented herein.

The rest of this paper is organised as follows. In Section 2, a preamble design technique for joint estimation of accurate timing and CID in UWAC systems with a high Doppler is proposed using a G-ZCS and a receiver processing scheme (decomposition and combining). In Section 3, the results of the performance evaluation of the existing and proposed techniques are presented. Computational complexity is compared in this section. Finally, in Section 4, the conclusions are presented.

\section{Preamble Design Technique Using a G-ZCS}

Figure 1 illustrates a downlink frame structure and the receiver processing for an OFDM-based UWAC system. A frame consists of a detection preamble, synchronisation preamble, and OFDM symbols for data burst. In the proposed technique, the detection preamble is used for joint estimation of timing and CID by correlating the received signal 
with a bank of locally generated preambles. Once the timing and CID are detected, the Doppler scale factor and the CFO are estimated using a repetitive pattern of a ZC sequence owing to its good correlation property in the time and frequency domains. The Doppler scaling effect on the received signal is compensated by a resampling process using the estimated Doppler scale factor. Coarse and fine CFO synchronisations are performed using eight-repetitive and two-repetitive patterns of a ZC sequence, respectively. A time-varying channel is estimated by the pilots inserted in each OFDM symbol. Finally, the transmitted data are recovered using the frequency-domain equalisers obtained using the pilots. In this paper, we focus on accurate timing and CID estimation in UWAC systems with a high Doppler because many techniques for $\mathrm{CFO}$ and Doppler scale estimation in underwater acoustic communication systems are already available $[43,48]$.

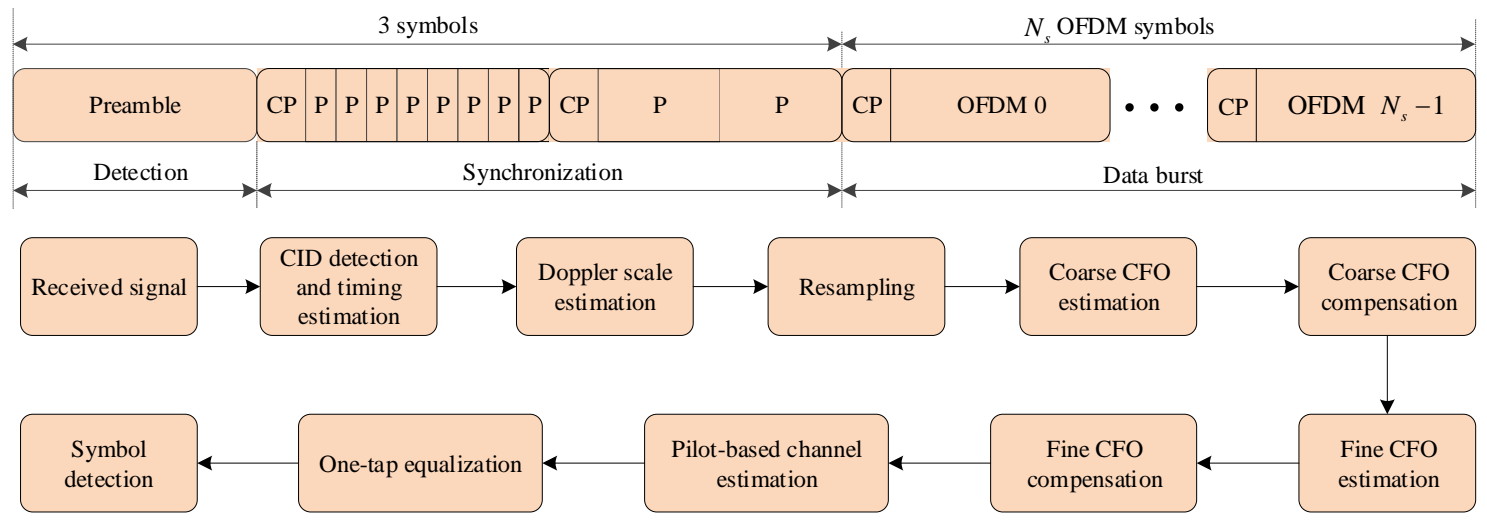

Figure 1. Downlink frame structure and receiver processing.

To jointly estimate the timing and CID in OFDM-based UWAC systems with a high Doppler, we propose a preamble design technique in this section. The ZC sequence is defined as [41]

$$
x_{p}(n)= \begin{cases}e^{j \pi p n(n)(n+1) / M} & \text { Odd Length } \\ e^{j \pi p n^{2} / M} & \text { Even Length } \\ e^{j \pi p n(n+M \bmod 2) / M} & \text { Prime Length }\end{cases}
$$

where $p$ and $M$ are the root index of the ZC sequence and the number of samples in the sequence, respectively. The root index $p$ is an integer that ranges from 1 to $M-1$. The ZC sequences are perfect sequences with an ideal autocorrelation function. When $M$ is odd, the cross-correlation function is given by $1 / \sqrt{M}$ using Gauss sums. In this paper, G-ZCS is defined as a $\mathrm{ZC}$ sequence with a root index expressed as a rational number ranging from $-\lceil M / 2\rceil$ to $\lfloor M / 2\rfloor$ except zero. The root index is generalised to a rational number with a different range. The G-ZCS can be decomposed into multiple short sequences consisting of other ZC sequences. The short sequence of the G-ZCS with the root index $p$ in (1) is defined as

$$
x_{p, q}(n)=e^{-j \pi \kappa_{p}\left(\alpha_{q}+n\right)^{2} / M_{p}},
$$

where $\kappa_{p}, M_{p}$, and $\alpha_{q}$ are $M_{p} /(M /|p|),\lfloor M /|p|\rfloor$, and $\operatorname{round}(q(M /|p|))$, respectively. Here, $q$, round (.), and $\lfloor$.$\rfloor denote the index of short sequence, round operation, and floor oper-$ ation, respectively. The root index $p$ is associated with the UWBS CID, and the integer $q$ ranges from 0 to $\lfloor|p|\rfloor$. The short sequence can be viewed as another G-ZCS with a root index $\kappa_{p}$. Here, $\left|\kappa_{p}\right|$ is 1 or almost equal to 1 . The length of the short sequence $M_{p}$ becomes 1 when $p$ has a value close to $M-1$. Thus, a value close to $M-1$ cannot be used for the root index in the preamble design. However, because $x_{p=M-a}(n)$ is same as $x_{p=-a}(n)$ in (1), $M-a$ can be replaced by $-a$, thus extending the range of available root indices. Therefore, the range of the root index in the G-ZCS is defined from $-\lceil M / 2\rceil$ to $\lfloor M / 2\rfloor$ except zero.

A UE/SN in a UWAC system receives preambles consisting of G-ZCS from neighbouring UWBSs with different $p$ values, and it detects the serving UWBS and corresponding 
timing estimate. The UE/SN estimates the timing and CID by correlating the received signal with the reference G-ZCSs. The correlation between the short sequences in (2) in the presence of Doppler is given by

$$
\begin{aligned}
& \widehat{R}_{\delta, v_{p}, k}^{p_{c}, p, q}=\sum_{n=0}^{M_{p}-1} e^{j \pi \kappa_{p}\left(\alpha_{q}+n\right)^{2} / M_{p}} e^{j 2 \pi v_{p} n / M_{p}} e^{-j \pi\left(p_{c} / p\right) \kappa_{p}\left(\alpha_{q}+n+\delta\right)^{2} / M_{p}} e^{j 2 \pi k n / M_{p}} \\
& =e^{j \pi\left(1-\left(p_{c} / p\right)\right) \kappa_{p}\left(\alpha_{q}\right)^{2} / M_{p}} e^{-j 2 \pi\left(p_{c} / p\right) \kappa_{p} \alpha_{q} \delta / M_{p}} e^{-j \pi\left(p_{c} / p\right) \kappa_{p}(\delta)^{2} / M_{p}} \\
& \quad \times \sum_{n=0}^{M_{p}-1} e^{j \pi\left(1-\left(p_{c} / p\right)\right) \kappa_{p}(n)^{2} / M_{p}} e^{j 2 \pi\left(\left(1-\left(p_{c} / p\right)\right) \kappa_{p} \alpha_{q}+v_{p}-\left(p_{c} / p\right) \kappa_{p} \delta+k\right) n / M_{p}}
\end{aligned}
$$

where $p_{c}$ is the root index of the signal received from the UWBS with CID $c$, and $p$ is the reference root index. If $p$ is equal to $p_{c}$, then (3) becomes an autocorrelation. The correlation between the short sequences of G-ZCS with root index $p$ in the presence of Doppler is given in (1). The first and third terms inside the sum operation represent the locally generated G-ZCS and received G-ZCS. The second term inside the sum operation represents the Doppler effect. The equation (1) can be considered a discrete-time domain version of ambiguity function where the received signal with timing offset is correlated with the transmitted signal in the presence of Doppler. Here, $v_{p}$ and $\delta$ denote the CFO caused by the Doppler shift and sample offset, respectively. Moreover, $v_{p}$ is given by $v\left(M_{p} / M\right)$, where $v$ is a normalised CFO for the ZC sequence with length $M$ in (1). Thus, $v_{p}<v$ when $p>1$, implying that the effect of Doppler shift on the short sequence is reduced. From (3), it can be observed that the correlation operation can be performed using inverse discrete Fourier transform because the product of ZC sequences with different time offsets is represented as a linear phase rotation when $p_{c}$ and $p$ are the same. When $p_{c}$ and $p$ are the same, the autocorrelation in the presence of Doppler shift is expressed as follows.

$$
\begin{aligned}
\widehat{R}_{\delta, v_{p}, k}^{p_{c}, p, q} & =e^{-j \pi \kappa_{p}\left(2 \alpha_{q} d+(\delta)^{2}\right) / M_{p}} \sum_{n=0}^{M_{p}-1} e^{-j 2 \pi\left\{\kappa_{p} \delta-v_{p}\right\} n / M_{p}} e^{j 2 \pi k n / M_{p}} \\
= & e^{-j \pi \kappa_{p}\left(2 \alpha_{q} \delta+(\delta)^{2}\right) / M_{p}} e^{j \pi\left(k-\kappa_{p} \delta+v_{p}\right)\left(M_{p}-1\right) / M_{p}} \frac{\sin \left(\pi\left(k-\kappa_{p} \delta+v_{p}\right)\right)}{\sin \left(\pi\left(k-\kappa_{p} \delta+v_{p}\right) / M_{r}\right)}
\end{aligned}
$$

When $p_{c}$ and $p$ are different, the cross-correlation in (3) can be expressed as follows.

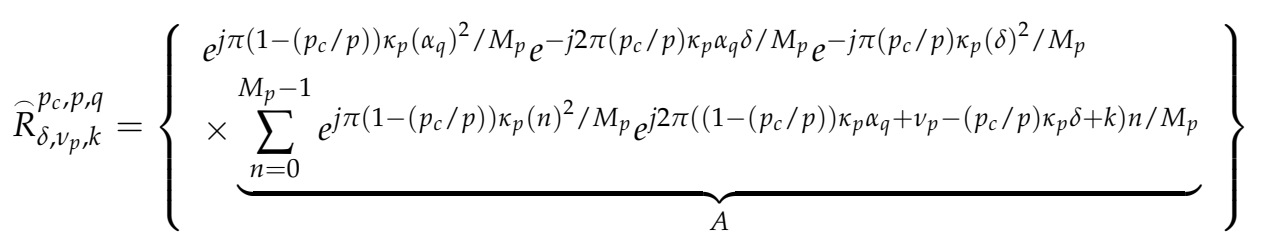

We define $T$ and $T_{S}(=T / M)$ as the symbol duration of the preamble and sampling period, respectively. When the sampling period $T_{S}$ approaches zero, the term $A$ in (5) can be approximated using an integral form as follows.

$$
\begin{aligned}
& \lim _{T_{s} \rightarrow 0} \sum_{n=0}^{M_{p}-1} e^{j \pi\left(\left(1-p_{c} / p\right) \kappa_{p} \frac{W}{T_{p}}\left(n T_{s}\right)^{2}\right.} e^{j 2 \pi\left\{\left(1-\left(p_{c} / p\right)\right) \kappa_{p} \alpha_{q}+v_{p}-\left(p_{c} / p\right) \kappa_{p} \delta+k\right\} n T_{s} / T_{p}} \\
& \simeq \frac{1}{T_{p}} \int_{0}^{T_{p}} e^{j \pi\left(1-p_{c} / p\right) \kappa_{p} W(t)^{2} / T_{p}} e^{j 2 \pi\left\{\left(1-\left(p_{c} / p\right)\right) \kappa_{p} \alpha_{q}+v_{p}-\left(p_{c} / p\right) \kappa_{p} \delta+k\right\} t / T_{p}} d t \\
& =\frac{1}{T_{p}} \int_{0}^{T_{p}} e^{j \pi \omega_{q}^{p_{c}, p}(t)^{2} / T_{r}} e^{j 2 \pi \gamma_{q, k}^{p_{c}, p} t} d t
\end{aligned}
$$

where $\omega^{p_{c}, p}=\left(1-p_{c} / p\right) \kappa_{p} W$ and $\gamma_{q, k}^{p_{c}, p}=\left(\left(1-\left(p_{c} / p\right)\right) \kappa_{p} \alpha_{q}+v_{p}-\left(p_{c} / p\right) \kappa_{p} \delta+k\right) \%_{p} /$ $\left(T_{p}\right)$. Here, $W$ denotes the operational channel bandwidth. The duration of the short sequence $T_{p}$ is given by $M_{p} T_{s}$. Using the Fresnel integrals, we can rewrite (6) as 


$$
\begin{aligned}
& A_{f}^{\omega^{p_{c}, p}}=\left\{\begin{array}{c}
\left(\frac{1}{T} \int_{0}^{T} e^{j \pi\left|\omega^{p_{c}, p}\right|(t)^{2} / T} e^{-j 2 \pi f t} d t\right)^{*}, \quad \omega^{p_{c}, p}<0 \\
\frac{1}{T} \int_{0}^{T} e^{j \pi\left|\omega^{p_{c}, p}\right|(t)^{2} / T} e^{j 2 \pi f t} d t, \quad \omega^{p_{c}, p}>0
\end{array}\right. \\
& =\frac{1}{T} \sqrt{\frac{T}{2\left|\omega^{p_{c}, p}\right|}} e^{j \pi\left(T_{p} /\left|\omega^{p_{c}, p}\right|\right)(f)^{2}}\left(C\left(x_{f, 0}^{\omega^{p_{c}, p}}\right)+C\left(x_{f, 1}^{\omega^{p_{c}, p}}\right)+j\left(\operatorname{sign}\left(\omega^{p_{c}, p}\right)\right)\left(S\left(x_{f, 0}^{\omega_{p_{c}, p}}\right)+S\left(x_{f, 1}^{\omega^{p_{c}, p}}\right)\right)\right)
\end{aligned}
$$

where $x_{f, 0}^{\omega^{p_{c}, p}}=\sqrt{\frac{2\left|\omega^{p_{c}, p}\right|}{T}}\left(-\frac{T}{\omega^{p_{c}, p}} f\right)$ and $x_{f, 1}^{\omega^{p_{c}, p}}=\sqrt{\frac{2\left|\omega^{p_{c}, p}\right|}{T}}\left(T+\frac{T}{\omega^{p_{c}, p}} f\right)$. Here, $C$ and $S$ denote the cosine Fresnel integral and sine Fresnel integral, respectively. The notation $f$ denotes $\gamma_{q, k}^{p_{c}, p}$ in (6). The notation $f$ is used because of the similarity between (7) and the Fourier transform. From the results in (6) and (7), we can obtain an approximate form of the cross-correlation of the short sequence, given as follows.

$$
\tilde{A}_{f}^{\omega^{p_{c}, p}}=\sum_{n=0}^{M_{p}-1} e^{j \pi\left(\omega^{p_{c}, p} / W\right)(n)^{2} / M_{p}} e^{j 2 \pi f n T_{s}} \approx \sum_{s=-\left\lceil\omega^{p_{c}, p} / W\right\rceil}^{1} A_{f+s W}^{\omega^{p_{c}, p}, W}
$$

When the correlation is obtained with a short sequence, the performance (signal detection and timing estimation) is not desirable owing to its short length. The performance loss can be compensated using the repetition property of the $\mathrm{ZC}$ sequence. The detection probability can be increased without sacrificing its robustness to Doppler shift by combining the correlation results obtained from short sequences. For example, Figure 2 shows the instantaneous frequency of a G-ZCS when $W, T_{S}, M$, and $p$ are set to $5 \mathrm{kHz}, 2 \mathrm{~ms}, 625$, and 5 , respectively. In this case, the symbol duration of the preamble, $T$, is $0.1250 \mathrm{~ms}$, as shown in Figure 2. Here, the instantaneous frequency is the time derivative of the phase term in (1). From Figure 2, it can be observed that the short sequence with length $M_{p}$ (125) is repeated $p$ (5) times within the sequence length $M$ (625). The correlation result after combining the short sequences is given as follows.

$$
\widehat{R}_{\delta, v_{p}, k}^{p_{c}, p}=\sum_{q=0}^{\lfloor p\rfloor-1} \widehat{R}_{\delta, v_{p}, k}^{p_{c}, p, q}\left|\approx \sum_{q=0}^{\lfloor p\rfloor-1}\right| \tilde{A}_{f=\gamma_{q, k}^{p_{c}, p}}^{\omega^{p_{c}, p}} \mid
$$

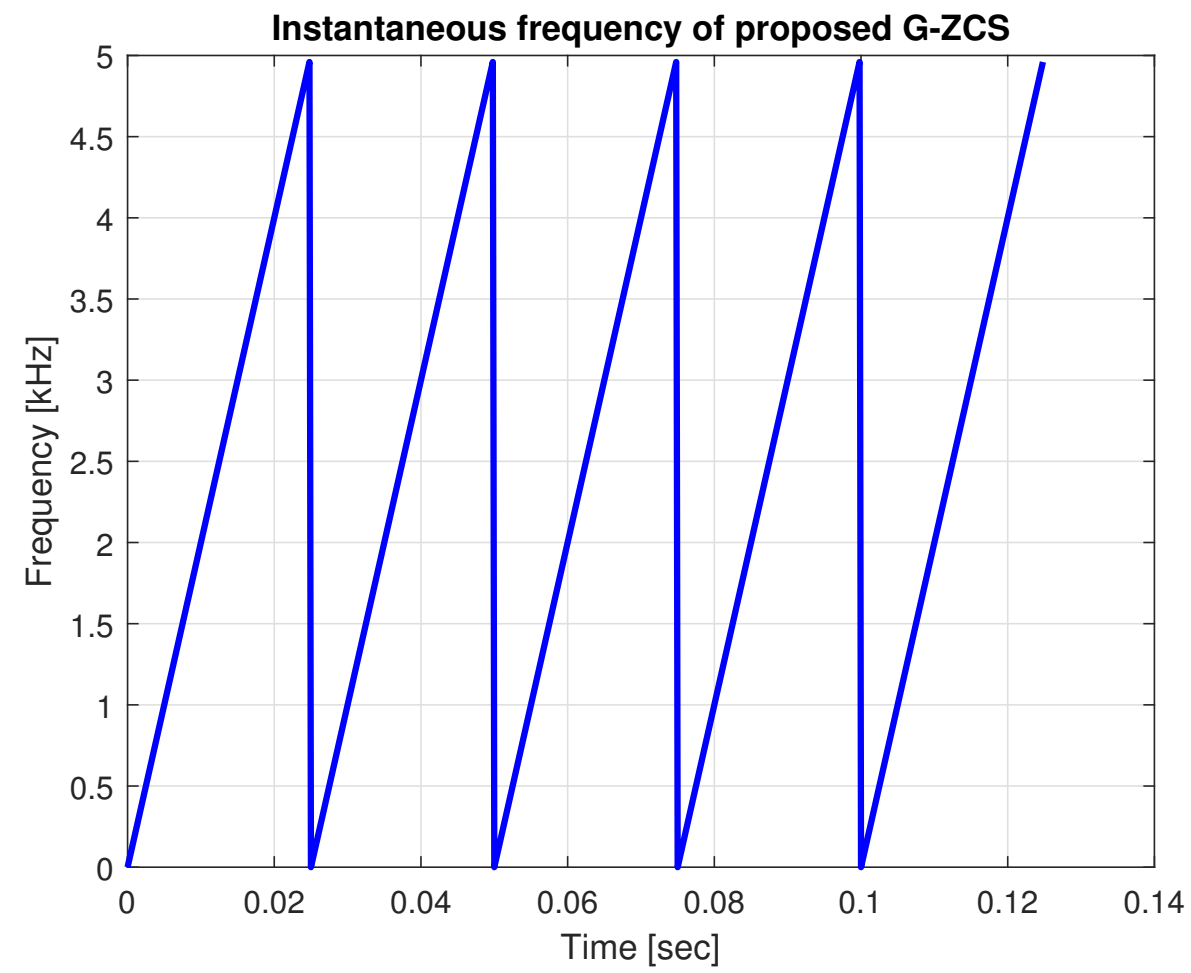

Figure 2. Instantaneous frequency of proposed G-ZCS. 
Here, a non-coherent combining is used to reduce the effect of phase rotation in (5) caused by the Doppler shift. The property of the ZC sequence is used in deriving (9); the $\mathrm{ZC}$ sequence with a non-zero $\delta$ is represented by the product of the ZC sequence and its phase rotation. Figure 3 shows the decomposition and combining $(D \& C)$ process for the proposed G-ZCS.

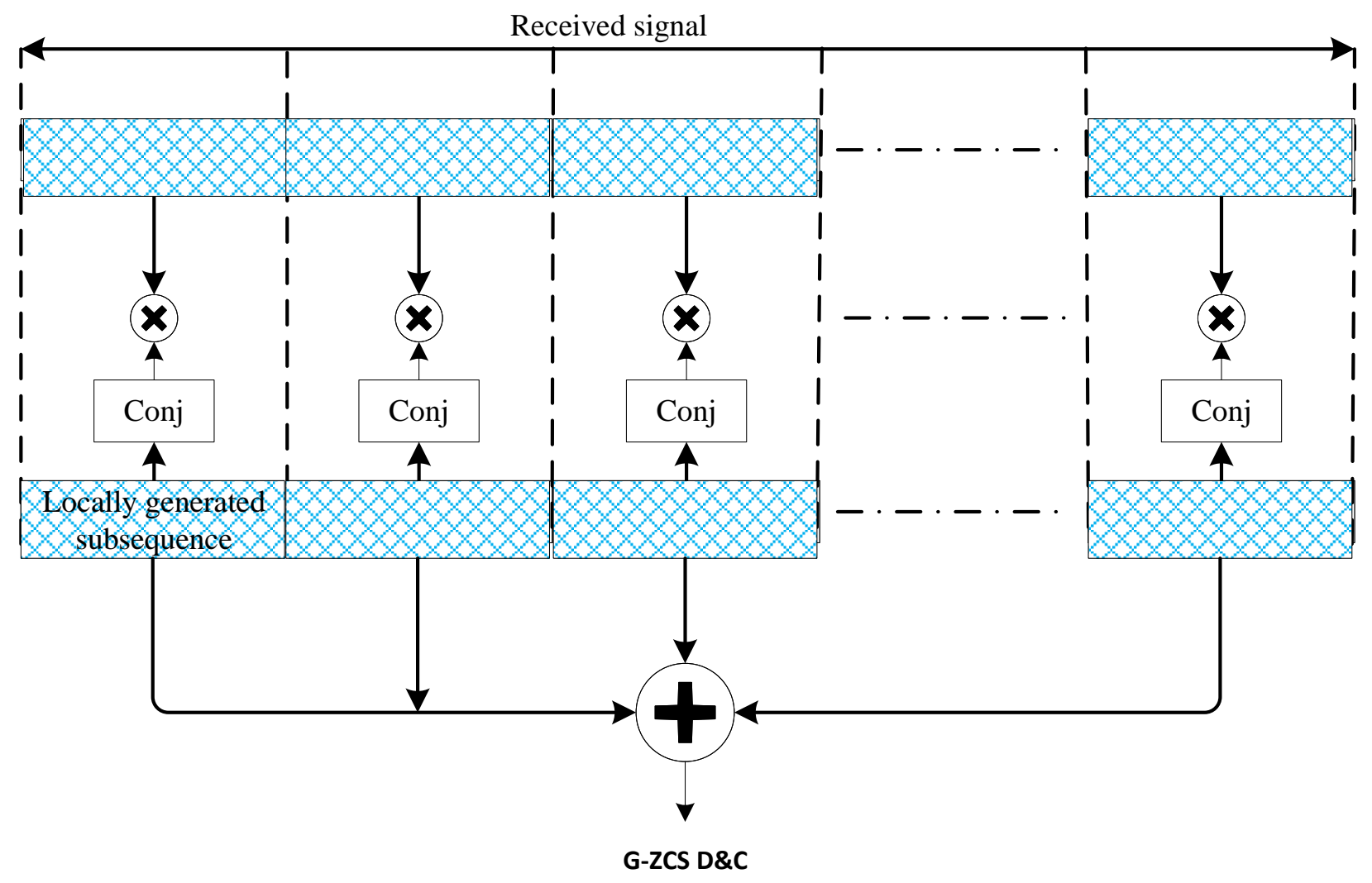

Figure 3. Decomposition and combining process for proposed G-ZCS.

Figure 4 shows an example of autocorrelation result of a G-ZCS. In the figure, $M$, $M_{p}, \delta, p_{c}, v_{p}$, and $v$ are set to $625,250,10,2.5,0.6$, and 1.5, respectively. The peak of the autocorrelation occurs at the $23^{\text {rd }}$ sample when the G-ZCS with length $M$ ('G-ZCS without $D \& C^{\prime}$ ) is used. A large (13) sample offset occurs owing to the frequency offset $v$. However, the peak occurs at the correct position $(\delta=10)$ when G-ZCS with D\&C is used. The simulation results are almost identical to the analytical results obtained by (4) and (9). Thus, the Doppler effect on timing estimation can be significantly reduced by the proposed sequence.

Figure 5 shows an example of the cross-correlation results of the proposed G-ZCS. In Figure $5 a, p_{c}$ is set to 10 . The value of $p$ ranges from -20 to 20 , with $\Delta p$ equal to 0.5 . Here, $\Delta p$ denotes the step size between adjacent root indices. In Figure 5a, 'G-ZCS without $D \& C^{\prime}$ represents the case where cross-correlation is obtained using a short sequence. The analytical results of 'G-ZCS with D\&C' are obtained using (8) and (9) to show the maximum cross-correlation value of the proposed G-ZCS after the combining process. From Figure 5a, it can be observed that the analytical result agrees well with the simulation results. Additionally, the cross-correlation value of the G-ZCS without D\&C is considerably larger than that of G-ZCS with D\&C. When $p$ is 10 , the maximum correlation value becomes 1 in both cases because the CID is matched $\left(p=p_{c}\right)$. In this case, the correlation operation becomes an autocorrelation. When $p$ is not 10 , the maximum correlation value of the G-ZCS with D\&C is less than 0.2. When $p$ is close to 20, it becomes 0.2. This is because the maximum correlation value increases as the sequence length decreases. Although not shown in Figure 5a, the maximum correlation value increases significantly when the 
step size between different $p$ values is less than 0.5 . Thus, $\Delta p$ should be set to a value greater than 0.5 . Figure $5 b, c$ shows examples of cross-correlation values for the proposed G-ZCS as a function of delay (samples). Here, $p_{c}, p, v_{p}$, and $M_{p}$ are set to 10, 3, 0, and 208 , respectively. When $p$ is 3 , the number of short sequences is 3 and length becomes 208 . From Figure $5 b, c$, it can be seen that the analytical results ((8) and (9)) match well with the simulation results. Furthermore, the maximum cross-correlation value of G-ZCS without $D \& C$ as shown in Figure $5 b$ is 0.13 , whereas that of G-ZCS with D\&C Figure $5 c$ is 0.083 .

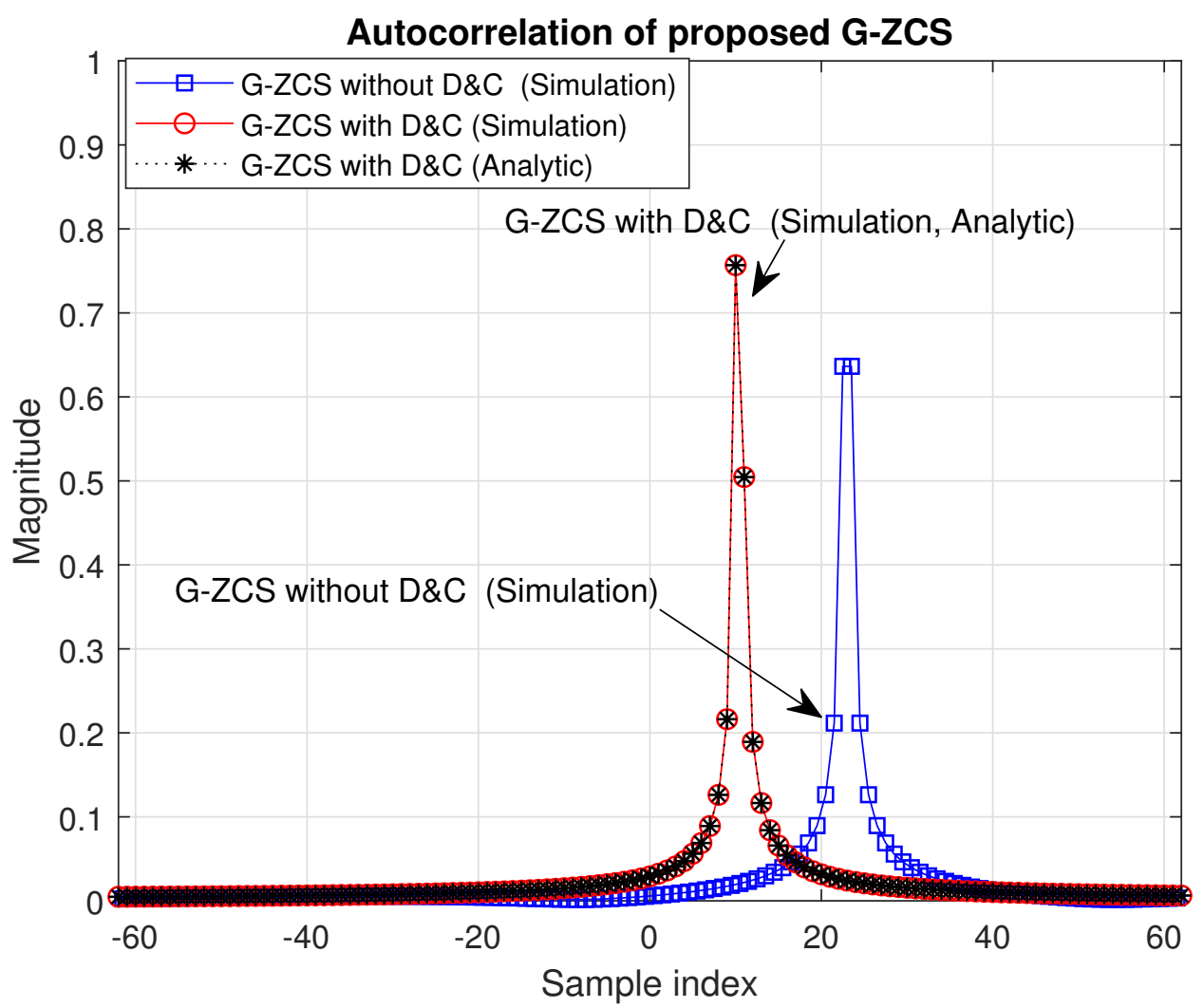

Figure 4. Autocorrelation result of the proposed G-ZCS ( $p_{c}=2.5, M=625, v=1.5$, and $\left.\delta=10\right)$.

Next, the relationship between the G-ZCS and conventional LFM waveform is described. The conventional LFM waveform is defined as $e^{j \pi \omega(t)^{2} / T} e^{j 2 \pi f t}$, where $T, \omega$, and $f$ denote the symbol duration, frequency sweeping parameter, and starting frequency, respectively. When $\omega$ is positive, the waveform is upchirp; if $\omega$ is negative, it is a downchirp. The maximum value of $\omega$ in the LFM waveform is the system bandwidth $(W)$. A discretised version of the LFM waveform can be obtained by sampling the waveform with the sampling period $T_{s}$ as follows.

$$
x_{L F M, \omega, M}(n)=e^{j \pi \omega\left(n T_{s}\right)^{2} / T} e^{j 2 \pi f n T_{s}}=e^{j \pi \omega T_{s}(n)^{2} / M} e^{j 2 \pi \tilde{f} n / M}
$$

where $T=M T_{s}$. The discretised version of the LFM waveform in (10) is similar to the G-ZCS $x_{p}(n)$ in (1). The terms, $\omega T_{s}$ and $\tilde{f}$, in (10) correspond to the root index $p$ and $(p / 2)\left(M_{\% 2}\right) / T$ in (1), respectively. The maximum value of $p$ is 1 because the parameter $|\omega|$ in the LFM waveform must be equal to or less than $W\left(\approx 1 / T_{s}\right)$. The term $\omega T_{S}$ in the LFM waveform (root index $p$ in the G-ZCS) is a rational number less than 1. Thus, the G-ZCS can be viewed as a generalised version of the LFM waveform by extending $|\omega|$ to be larger than $W$. The range of $p$ in the G-ZCS is a rational number from $-\lceil M / 2\rceil$ to $\lfloor M / 2\rfloor$ except zero. Thus, the G-ZCS becomes the conventional LFM waveform when the range of $p$ is limited between -1 to 1 . In addition, the short sequence $x_{p}, q(n)$ in (2) can be considered as the sampled version of the LFM waveform with $\omega$ equal to $\kappa_{p} / T_{s}$. The short sequence 
can be viewed as an LFM waveform with $|\omega|$ equal to or less than the system bandwidth $\left(\approx 1 / T_{s}\right)$ because $\left|\kappa_{p}\right| \leq 1$. The proposed G-ZCS shown in Figure 2 can also be interpreted using the generalised version of the LFM waveform with $\omega$ ranging from $-\lceil M / 2\rceil / T_{S}$ to $\lfloor M / 2\rfloor / T_{s}$. In Figure 2, the system bandwidth $(W)$ is set to $5 \mathrm{kHz}$. The parameter $\omega$ is set to five times the system bandwidth $(p W)$. If the parameter $\omega$ is set to the system bandwidth $W\left(\approx 1 / T_{s}\right)$ as in the case of the conventional LFM waveform, a significant shift in the position of the correlation peak occurs when Doppler shift exists. The short sequence in the G-ZCS can be viewed as a conventional LFM signal with a duration equal to $M_{p} T_{s}$ and $\omega$ equal to $W$. The short sequence in Figure 2 can be interpreted as an upchirp signal with $\omega$ equal to system bandwidth $(5 \mathrm{kHz})$. As the upchirp signal with $\omega$ is one-fifth of the length of the original waveform, it is five times more insensitive to the Doppler shift. However, a performance loss occurs owing to the shorter length of the upchirp signal with a duration $M_{p} T_{s}$. The performance can be improved by combining five upchirp signals without decreasing their robustness to Doppler shift.

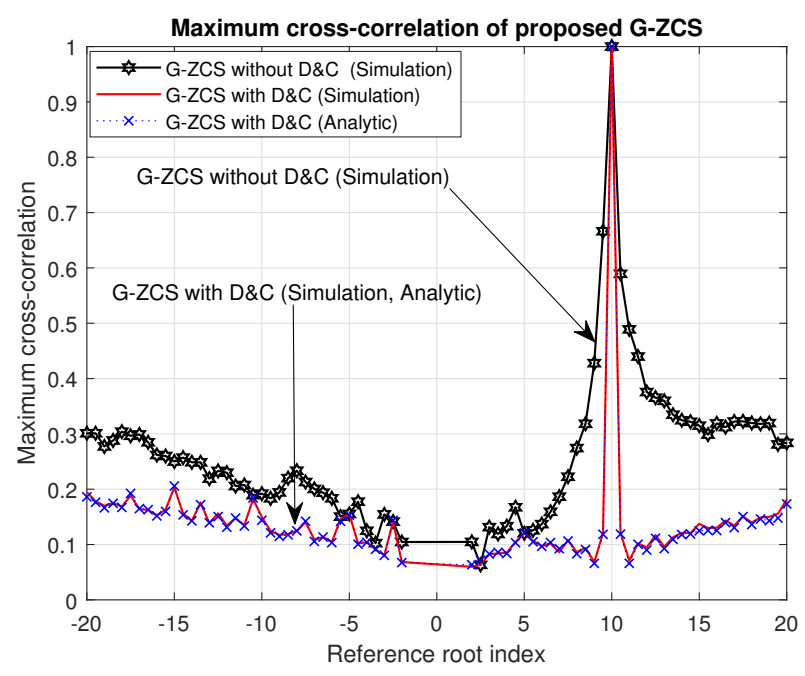

(a)

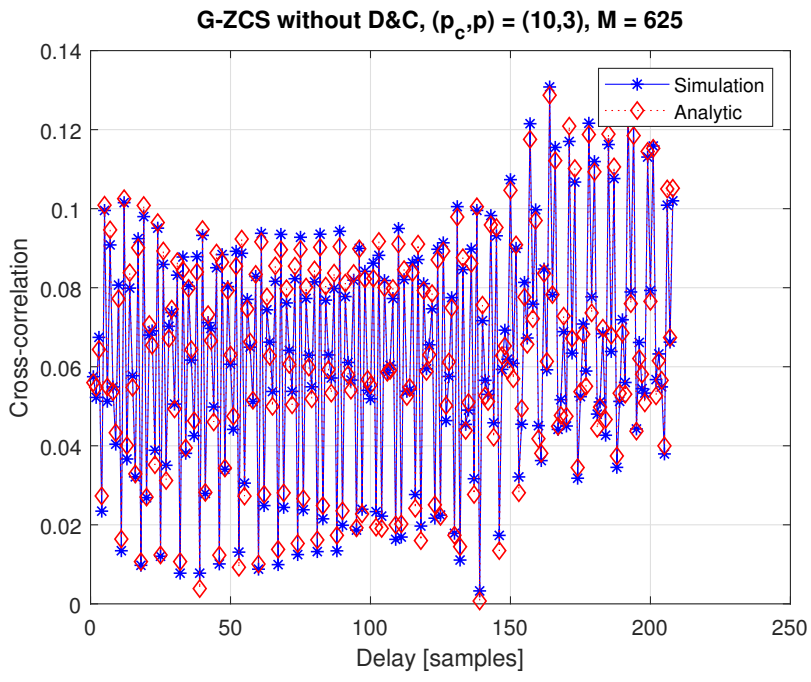

(b)

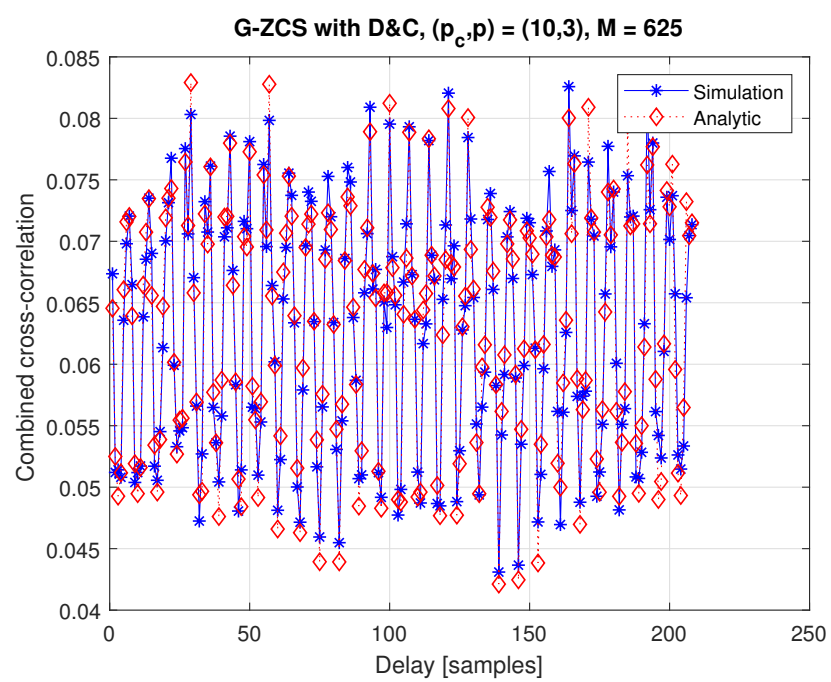

(c)

Figure 5. (a) Maximum cross-correlation of the proposed G-ZCS with different $p$ values. (b) Cross-correlation value of the proposed G-ZCS as a function of delay (samples). (c) Cross-correlation value of the proposed G-ZCS as a function of delay (samples). 
In the proposed technique, the preamble is generated in the transmitter (UWBS) using the G-ZCS with a root index corresponding to its CID, as given in (1). In the receiver (UE/SN), the received signal is correlated with all possible reference signals (ZC short sequences) and combined, as given in (9). Furthermore, the timing and CID are obtained by determining the peak position and root index maximizing the correlation function, respectively. In the proposed technique, there is a trade-off between the cross-correlation levels and the number of possible preambles (CIDs) when selecting the value of the root index $p$ and $\Delta p$. We can reduce the cross-correlation (intercell interference) between the preambles by increasing the value of $\Delta p$. However, the number of possible CIDs decreases with an increase in $\Delta p$. For example, in Figure $5 \mathrm{a}, p$ ranges from -20 to 20 , with $\Delta p$ equal to 0.5 . If $\Delta p$ is smaller than 0.5 , the maximum cross-correlation value will increases significantly. By limiting the maximum value of $|p|$ to 20 , we can maintain the crosscorrelation level below 0.2. In addition, in the proposed technique, the minimum value of the root index must be selected considering the maximum Doppler frequency and timing accuracy required for the system. To avoid performance degradation caused by Doppler shift in timing estimation, we need to set $\left|v_{p}\right|\left(=|v|\left(\left\lfloor M /\left|p_{c}\right|\right\rfloor / M\right)\right)$ to a value considerably less than $0.5\left(\delta_{\max }+1\right)$. Here, $\delta_{\max }$ denotes the maximum timing offset. This implies that $|v| M /\left(\left|p_{c}\right| M\right)$ must be much smaller than $0.5\left(\delta_{\max }+1\right)$. Thus, the minimum value of $\left|p_{c}\right|$ must satisfy this condition: $2 v_{\max } /\left(\delta_{\max }+1\right)$. For example, when the exact timing $\left(\delta_{\max }=0\right)$ is required, the value of $v_{p}$ must be smaller than 0.5 . This implies that $\left|p_{c}\right|$ must be greater than $v / 0.5$. In other words, if $v$ is $1,\left|p_{c}\right|$ should be greater than 2 . If $v$ is $0.5,\left|p_{c}\right|$ should be greater than 1 . The case of $\left|p_{c}\right|=1$ corresponds to the conventional LFM waveform.

The LFM waveform is extensively used for monitoring/surveillance applications because the target needs to be detected in the presence of Doppler shift. However, the LFM waveform is not adequate for an accurate estimation of the timing in high Doppler environments because it cannot produce the correct timing. The ambiguity function (AF) is widely used to analyse the resolution, sidelobe behaviour, and ambiguities in both Doppler shift and delay (time shift) of a given waveform. The AF of the LFM waveform is given as a skewed version of the triangular ridge in delay-Doppler plane. From the AF of the LFM waveform, we can see that the LFM waveform is beneficial for surveillance applications because it can detect the target regardless of the Doppler shift. In contrast, for timing estimation in high Doppler environments, a waveform is considered beneficial if its AF can produce a high peak at the correct timing regardless of the amount of Doppler shift. Thus, the ideal AF for an accurate estimation of the timing will be 'a line' located on the horizontal (Doppler) axis. The AF of G-ZCS is given in (4). Figure $6 \mathrm{a}, \mathrm{b}$ show maximum magnitude and time shift, respectively, in the AFs of LFM-FT, LFM-FF, and G-ZCS, when the Doppler shift varies. Here, $M=1024, M_{p}=60, p=17$, and $\omega=W=5 \mathrm{kHz}$. Here, $\omega$ is set to the maximum available bandwidth $(W)$. From Figure $6 a$, it can be observed that the fluctuation of maximum magnitude is the largest in the case of LFM-FT. The fluctuation levels of LFM-FF and G-ZCS with D\&C are similar. From Figure $6 b$, it can be observed that the LFM-FT and LFM-FF produce high peaks at the incorrect timing when Doppler shift exists. Their AFs are skewed versions of triangular ridge in delay-Doppler plane. However, the proposed G-ZCS with D\&C produces high peaks at the correct timing regardless of the Doppler shift. The AF of G-ZCS with D\&C is given by 'a line' located on the horizontal axis, implying that the G-ZCS with D\&C can accurately detect timing regardless of the Doppler shift. 


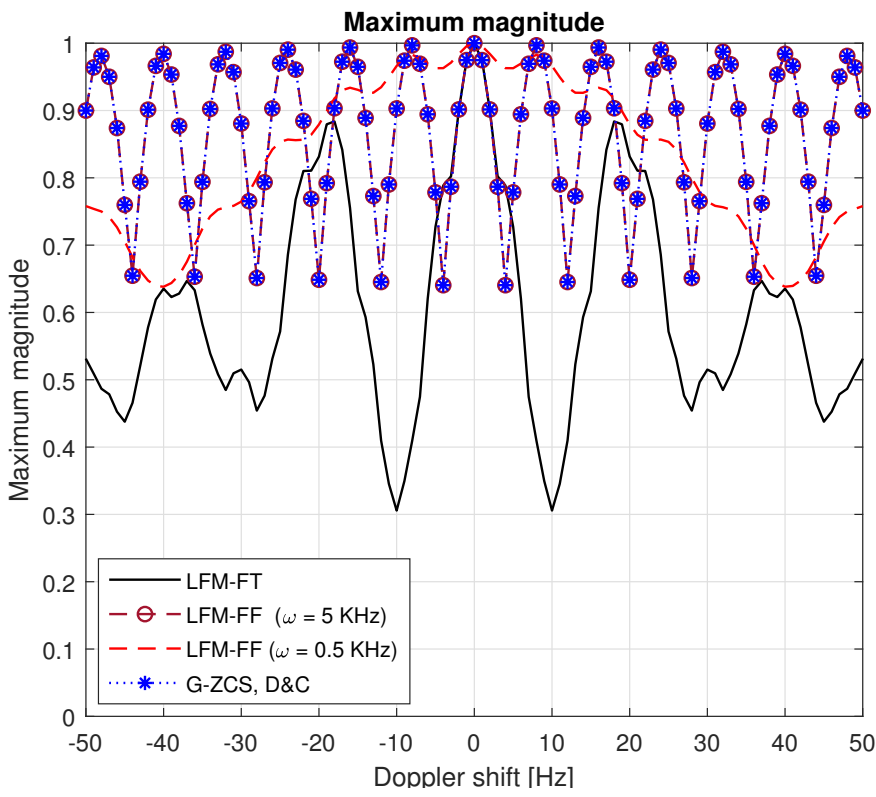

(a)

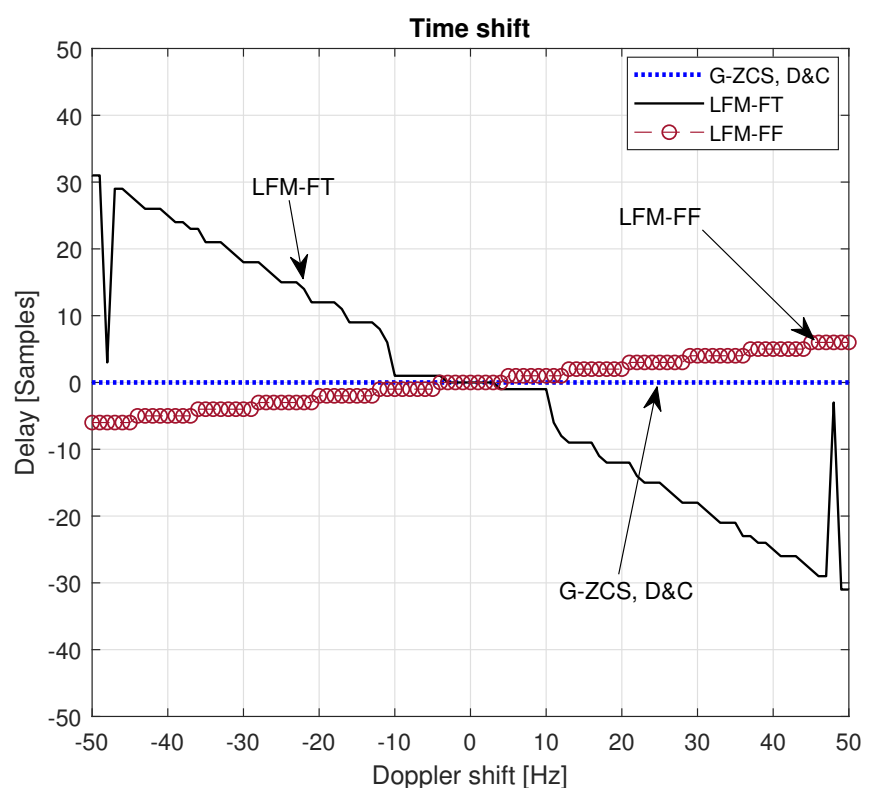

(b)

Figure 6. Maximum magnitude and time shift of ambiguity functions of previous and proposed sequences. (a) Maximum magnitude vs. Doppler shift. (b) Time shift vs. Doppler shift.

\section{Simulation}

In 2015, a new research study on UWAC systems was launched in Korea. Its ultimate goal was to deploy an UWAC system connected to a terrestrial cellular network. The experimental sites were located in the South Sea and West Sea of the Korean Peninsula. The project successfully completed the data transmission and downlink synchronisation parts using a transducer, hydrophone, and underwater modem, which were designed for this project.

Figure 7 shows the conceptual design of UWAC system whereas top view of three-cell model used for the simulation is shown in Figure 8. The performance of the proposed G-ZCS with D\&C is evaluated through computer simulations to decrease the receiver complexity and processing time. The cell radius $R_{c}$ was set to $5 \mathrm{~km}$. One UE was located at the boundary of cell 1. The distance between UE and UWBS1/UWBS2/UWBS3 was $4.5 / 5.14 / 5.39 \mathrm{~km}$. The path losses at these distances were $81.34 \mathrm{~dB}, 86 \mathrm{~dB}$, and $87.76 \mathrm{~dB}$ for UWBS1, UWBS2, and UWBS3, respectively. Thus, UWBS1 needs to be detected as a serving cell for the UE, whereas UWBS2 and UWBS3 are regarded as interfering cells. The frequency reuse factor was assumed to be 1 . The performance of the proposed G-ZCS with D\&C was compared with those of previous techniques (LFM-FT-CST and LFM-FF-CST). Exploiting the hexagonal geometry, we obtained the distance between the UE and each UWBS as follows.

$$
d=\frac{1}{2} \begin{cases}2 \sqrt{x_{0}^{2}+y_{0}^{2}}, & \text { UE - UWBS1 } \\ \sqrt{\left(3 R_{c}-2 x_{0}\right)^{2}+\left(\sqrt{3} R_{c}-2 y_{0}\right)^{2}}, & \text { UE - UWBS2 } \\ \sqrt{\left(3 R_{c}-2 x_{0}\right)^{2}+\left(\sqrt{3} R_{c}+2 y_{0}\right)^{2}}, & \text { UE - UWBS3 }\end{cases}
$$

Here, $x_{0}$ and $y_{0}$ denote the coordinate values of the point where the UE is located. Because the channel bandwidth was $5 \mathrm{kHz}$, the sampling rate was set to $5 \mathrm{kHz}$. The time difference of arrival (TDoA) normalised to the earliest arrival path is as follows: 0 sample $\{(4.5025 \mathrm{~km} / 1.5 \mathrm{~km})-(4.5025 \mathrm{~km} / 1.5 \mathrm{~km}) \times 5 \mathrm{kHz}\}$ for UWBS1, $2142 \mathrm{sam}-$ ples $\{(5.1452 \mathrm{~km} / 1.5 \mathrm{~km})-(4.5025 \mathrm{~km} / 1.5 \mathrm{~km}) \times 5 \mathrm{kHz}\}$ for UWBS2, and 2964 samples 
$\{(5.3918 \mathrm{~km} / 1.5 \mathrm{~km})-(4.5025 \mathrm{~km} / 1.5 \mathrm{~km}) \times 5 \mathrm{kHz}\}$ for UWBS3. Simulation parameters are summarised in Table 2. The large discrepancy in the TDoA was due to the low speed of the sound wave results in an asynchronous system even when all the UWBSs were capable of transmitting their signals synchronously.

Table 2. Simulation parameters.

\begin{tabular}{cc}
\hline Parameters & Values \\
\hline FFT size & 512 \\
Channel bandwidth & $5 \mathrm{kHz}$ \\
Subcarrier spacing & $9.76 \mathrm{kHz}$ \\
Carrier frequency & $24.5 \mathrm{kHz}$ \\
OFDM Symbol duration & $125 \mathrm{~ms}$ \\
Cell radius & $5 \mathrm{~km}$ \\
Distance (UE-UWBS1/ UWBS2/ UWBS3) & $4.5 / 5.14 / 5.39 \mathrm{~km}$ \\
Path loss (UE-UWBS1 / UWBS2/ UWBS3) & $81.34 / 86 / 87.76 \mathrm{~dB}$ \\
TDoA (UE-UWBS1/ UWBS2/ UWBS3) & $0 / 2142 / 2964 \mathrm{samples}$ \\
\hline
\end{tabular}

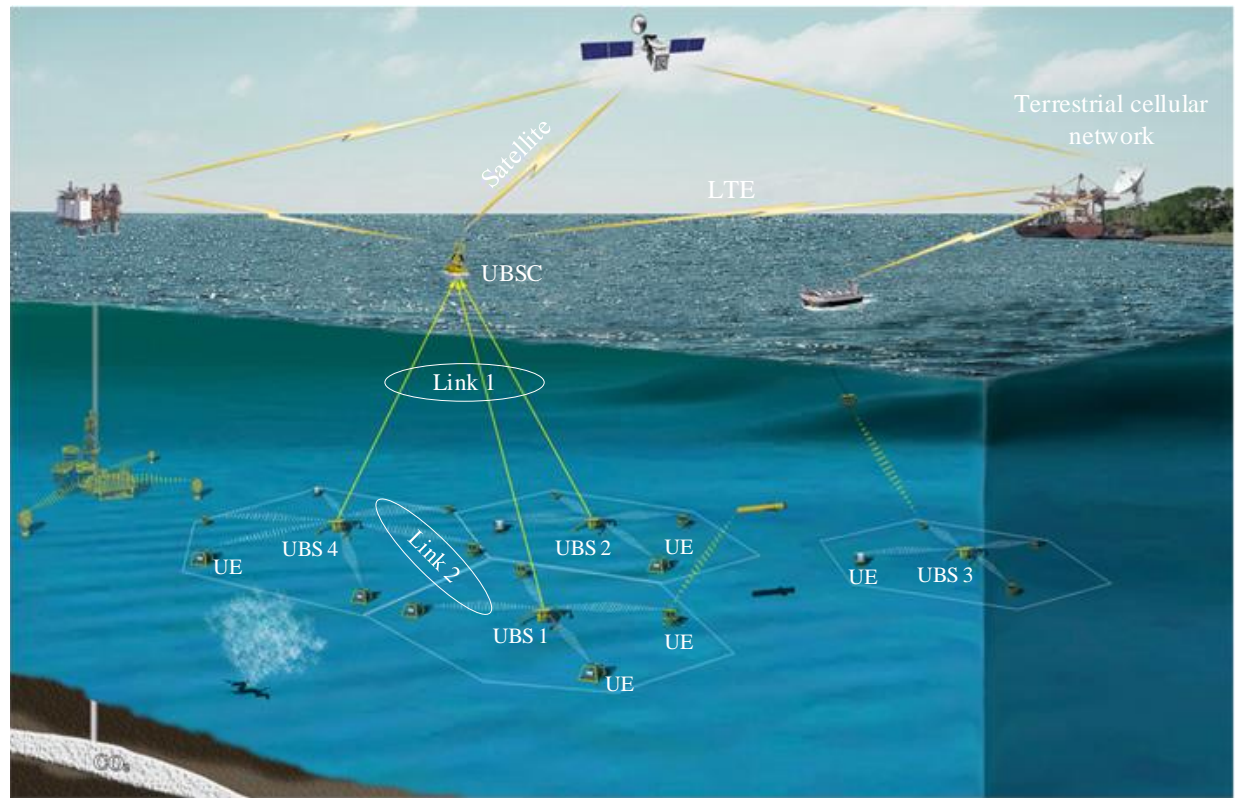

Figure 7. Design of UWAC cellular system.

For the simulation, two different channels were used: additive white Gaussian noise (AWGN) and multipath fading channels. The multipath fading channel is used to evaluate the performance of the proposed technique in underwater acoustic channels $[49,50]$. The multipath fading channel is generated by a Bellhop channel simulator, which is widely used for underwater acoustic communication. The parameters for the Bellhop channel simulator were set to the information provided in the field experimental site (distance: $2 \mathrm{~km}$, depth: $50 \mathrm{~m}$ ). Table 3 lists the multipath fading channel and the power delay profile generated by the simulator. The multipath fading channel consists of 12 paths, each experiencing Rician fading with different $K$ factors. The corresponding path delay and magnitude are listed in the table. A Doppler shift was applied to both the LOS $\left(f_{d L O S}\right)$ and NLOS $\left(f_{d N L O S}\right)$ components, which makes the channel time varying. 


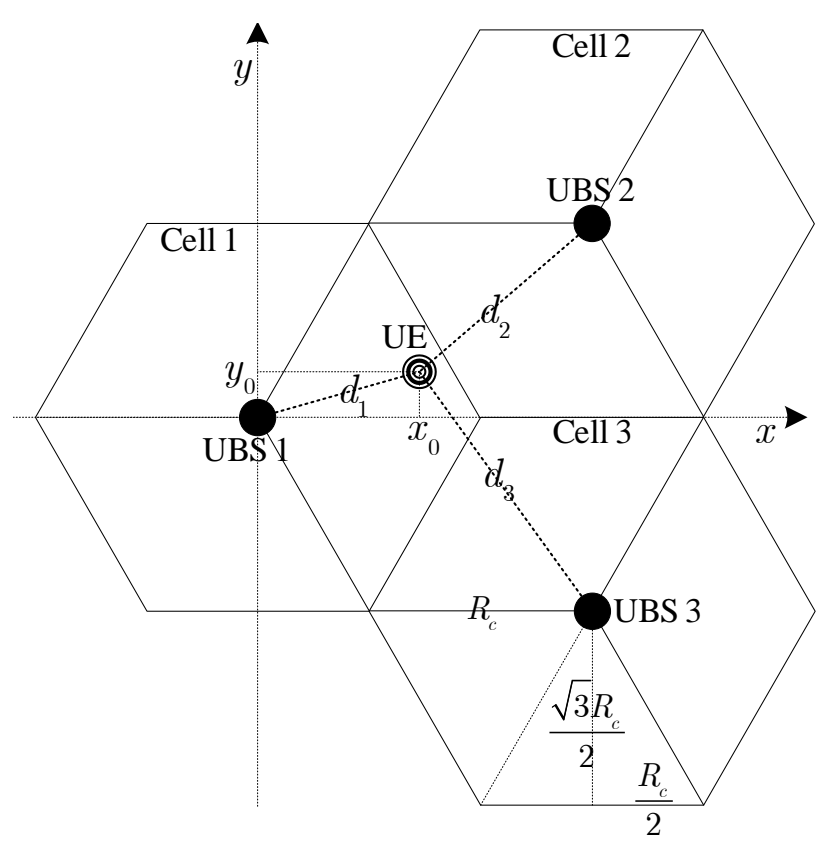

Figure 8. Top view of a typical UWAC system with 3 UWBSs.

Table 3. Multipath channel generated by Bellhop channel model.

\begin{tabular}{ccc}
\hline Path Delay [ms] & Magnitude & K Factor [dB] \\
\hline 0 & 0.537 & 43.50 \\
0.06 & 0.580 & 52.48 \\
1.50 & 0.427 & 19.95 \\
1.56 & 0.267 & 119.31 \\
1.67 & 0.274 & 61.68 \\
6.33 & 0.183 & 12.59 \\
6.39 & 0.073 & 79.43 \\
6.61 & 0.0650 & 31.62 \\
6.68 & 0.0303 & 50.12 \\
14.39 & 0.0289 & 50.12 \\
14.50 & 0.0065 & 31.62 \\
14.78 & 0.0067 & 12.59 \\
\hline
\end{tabular}

Figures 9 and 10 compares the detection probabilities of the proposed technique (GZCS with D\&C) and previous techniques (LFM-FT-CST and LFM-FF-CST) for an AWGN and multipath fading (Bellhop) channel. Here, the detection is declared 'successful' when the detected peak position (timing) and corresponding CID are correct. In the proposed technique, root indices of three UWBSs are chosen among the candidate sets consisting of root indices ranging from -20 to 20 . The step size $\Delta p$ is set to 0.5 . The parameters of the previous techniques for three UWBSs are set as given in [47]. From Figure 9, the detection probabilities of the previous techniques (LFM-FT-CST and LFM-FF-CST) are observed to start degrading as the Doppler shift increases. When the Doppler shift is $3 \mathrm{~Hz}$ and SNR is $5 \mathrm{~dB}$, the detection probabilities of LFM-FT-CST and LFM-FF-CST become $50 \%$ and $60 \%$, respectively. When the Doppler shift is $20 \mathrm{~Hz}$, their detection probabilities reach zero because the position of correlation peak is shifted significantly from the correct timing. However, if only coarse timing and CID need to be detected in the presence of Doppler shift, the previous techniques can be used successfully. However, in this case, an additional preamble and receiver processing block are required to estimate fine timing, resulting in increased receiver complexity and processing time. In the case of proposed technique (GZCS with D\&C), the detection probability decreases slightly as the Doppler shift increases. However, no significant performance degradation occurs even at the Doppler shift of $20 \mathrm{~Hz}$. 
The detection probability of the proposed technique decreases slightly in multipath fading channel as shown in Figure 10. However, it can still reach $100 \%$ at the SNR of $-10 \mathrm{~dB}$ when the Doppler shift is $20 \mathrm{~Hz}$. Here, a Doppler shift of $20 \mathrm{~Hz}$ is considered because the average speed of AUVs is $1 \mathrm{~m} / \mathrm{s}$ [51]. A speed of $1 \mathrm{~m} / \mathrm{s}$ corresponds to a Doppler shift of $16.33 \mathrm{~Hz}$ in an underwater acoustic channel.

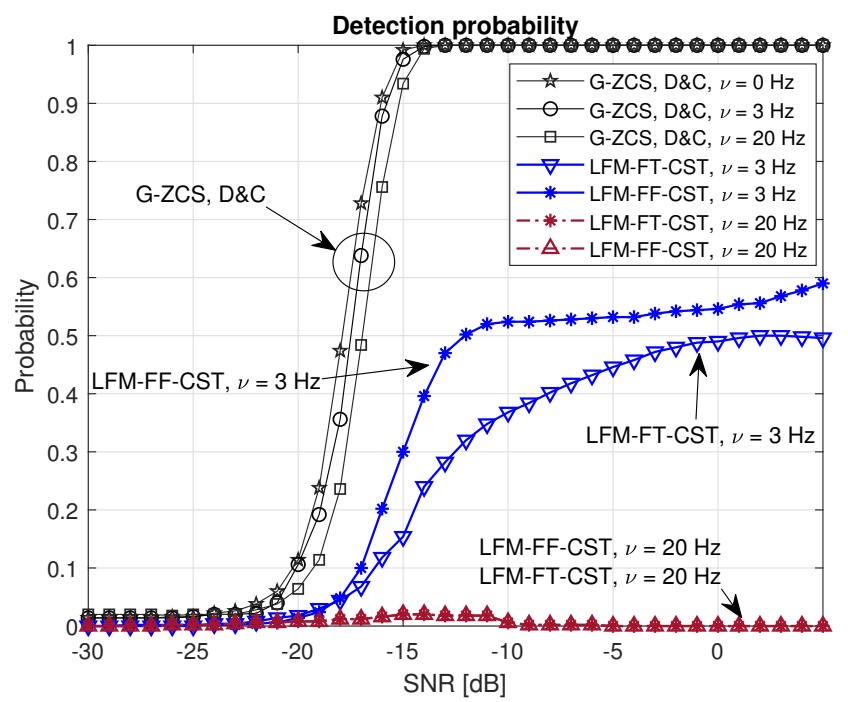

Figure 9. Detection probabilities of the proposed G-ZCS, D\&C, and conventional CSTs (AWGN).

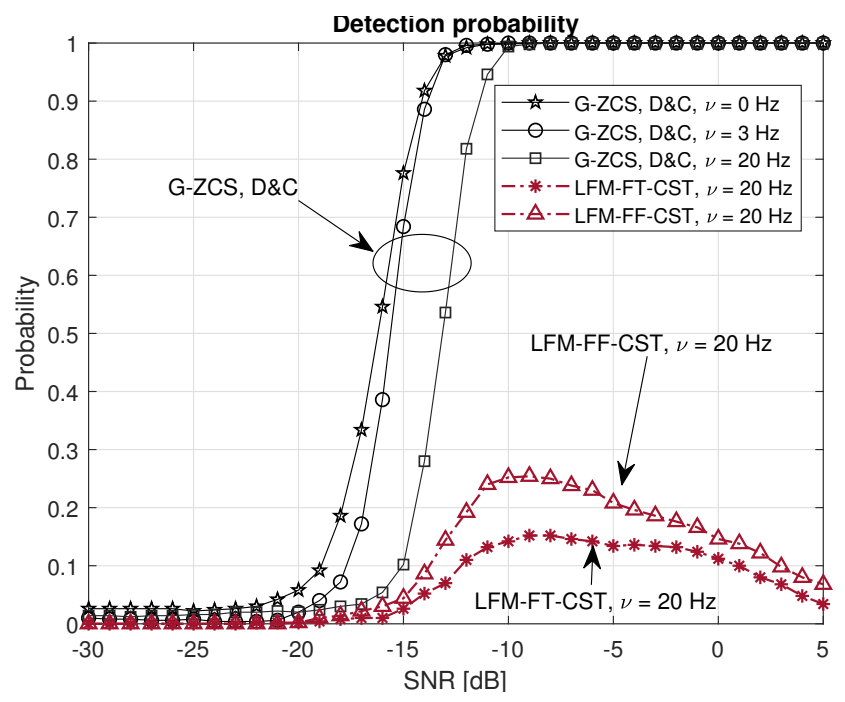

Figure 10. Detection probabilities of the proposed G-ZCS, D\&C, and conventional CSTs (Bellhop).

Computational Complexity

Table 4 shows the computation complexity required to estimate timing and CID for a UWAC system in terms of number of multiplications. Here, it is assumed that the number of neighboring UWBSs, denoted by $B$, is 20 . The number of samples in a short sequence of G-ZCS, denoted by $M_{p}$, is 125 . The short sequence is repeated $p(5)$ times in a symbol duration. Here, $p$ denotes the root index. Similarly, in the case of LFM-FT-CST and LFMFF-CST, the number of samples in a symbol duration, denoted by $M$, is 625 . The number of samples in a full sequence of G-ZCS is the same as M. As can be seen in Table 4, the computational complexities in both the previous and proposed techniques are the same. However, the proposed technique performs better than the previous technique, especially in high Doppler environments, as can be seen in Figures 9 and 10. 
Table 4. Computational complexity.

\begin{tabular}{ccc}
\hline Techniques & $\begin{array}{c}\text { Number of Complex } \\
\text { Multiplications }\end{array}$ & Example \\
\hline G-ZCS & $\sum_{q=1}^{p} M_{p, q} \times B$ & $\begin{array}{l}(125 \times 20)+(125 \times 20)+(125 \times 20) \\
+(125 \times 20)+(125 \times 20)=12,500\end{array}$ \\
\hline LFM-FT-CST, & $M \times B$ & $625 \times 20=12,500$ \\
LFM-FF-CST & $M$ & \\
\hline
\end{tabular}

\section{Conclusions}

In this paper, a preamble design technique for accurate timing estimation and CID detection in UWAC systems with a high Doppler is proposed using a G-ZCS. The autocorrelation and cross-correlation properties of the proposed G-ZCS were derived and shown to be consistent with the simulation results. The maximum cross-correlation level that determines the intercell interference between preambles can be significantly reduced by the proposed G-ZCS with D\&C. The selection rule for the root index in the G-ZCS is discussed considering the cross-correlation level, number of possible CIDs, maximum Doppler frequency, and timing accuracy. The simulation results also show that the existing techniques (LFM-FT-CST and LFM-FF-CST) produce a significant timing offset in highDoppler environments whereas the proposed sequence produces almost no time ambiguity. Finally, the proposed technique (G-ZCS with $D \& C$ ) is shown to be suitable for accurate timing estimation and CID detection in UWAC systems with a high Doppler. In this project, we successfully completed data transmission and synchronisation parts for fixed sensor nodes in a UWAC system constructed in the testbed (South Sea of Korean Peninsula). In the next project, we plan to evaluate the performance of the proposed technique (G-ZCS with D\&C) in the UWAC system using autonomous underwater vehicles (AUVs) with different velocities.

Author Contributions: Y.J.K., M.A. and T.H.I. developed the preamble design technique for timing and CID estimation for underwater acoustic cellular systems. Y.S.C. supervised the project. All authors have read and agreed to the published version of the manuscript.

Funding: This research was part of the project titled 'Development of Distributed Underwater Monitoring \& Control Networks', funded by the Ministry of Oceans and Fisheries, South Korea.

Conflicts of Interest: The authors declare no conflict of interest.

$\begin{array}{ll}\text { Acronyms } \\ \text { UWAC } & \text { Underwater acoustic cellular } \\ \text { CID } & \text { Cell identification } \\ \text { UE/SN } & \text { User equipment/sensor node } \\ \text { LFM } & \text { Linear frequency modulation } \\ \text { ZCS } & \text { Zadoff-Chu sequence } \\ \text { UWBS } & \text { Underwater base station } \\ \text { CFO } & \text { Carrier frequency offset } \\ \text { OFDM } & \text { Orthogonal frequency division multiplexing } \\ \text { CDMA } & \text { Code division multiple access } \\ \text { AUVs } & \text { Autonomous underwater vehicles } \\ \text { FFT } & \text { Fast fourier transform } \\ \text { LOS } & \text { Line of sight } \\ \text { NLOS } & \text { Non-line of sight } \\ \text { CP } & \text { Cyclic prefix } \\ \text { D\&C } & \text { Decomposition and combining } \\ \text { LFM-FT } & \text { LFM in time doomain } \\ \text { LFM-FT } & \text { LFM in frequency domian } \\ \text { CSTs } & \text { Cell searching techniques }\end{array}$


AWGN Additive white gaussian noise

G-ZCS Generalised ZCS

\section{References}

1. Sendra, S.; Lloret, J.; Jimenez, J.M.; Parra, L. Underwater acoustic modems. IEEE Sens. J. 2016, 16, 4063-4071. [CrossRef]

2. Yu, X.; Zhuang, X.; Li, Y.; Zhang, Y. Real-time observation of range-averaged temperature by high-frequency underwater acoustic thermometry. IEEE Access 2019, 7, 17975-17980. [CrossRef]

3. Qiu, J.; Xing, Z.; Zhu, C.; Lu, K.; He, J.; Sun, Y.; Yin, L. Centralized fusion based on interacting multiple model and adaptive Kalman filter for target tracking in underwater acoustic sensor networks. IEEE Access 2019, 7, 25948-25958. [CrossRef]

4. Akhoundi, F.; Minoofar, A.; Salehi, J.A. Underwater positioning system based on cellular underwater wireless optical CDMA networks. In Proceedings of the 26th Wireless and Optical Communication Conference (WOCC), Newark, NJ, USA, 7-8 April 2017.

5. Erol-Kantarci, M.; Mouftah, H.T.; Oktug, S. A survey of architectures and localization techniques for underwater acoustic sensor networks. IEEE Commun. Surv. Tutorials 2011, 13, 487-502. [CrossRef]

6. Rossi, P.S.; Ciuonzo, D.; Ekman, T.; Dong, H. Energy Detection for MIMO Decision Fusion in Underwater Sensor Networks. IEEE Sensors J. 2015, 15, 1630-1640. [CrossRef]

7. Cai, S.; Zhu, Y.; Wang, T.; Xu, G.; Liu, A.; Liu, X. Data collection in underwater sensor networks based on mobile edge computing. IEEE Access 2019, 7, 65357-65367. [CrossRef]

8. Tan, H.P.; Diamant, R.; Seah, W.K.G.; Waldmeyer, M. A survey of techniques and challenges in underwater localization. Ocean Eng. 2011, 38, 1663-1676. [CrossRef]

9. Akyildiz, I.F.; Pompili, D.; Melodia, T. Underwater acoustic sensor networks: Research challenges. Ad. Hoc. Netw. 2005, 3, 257-279. [CrossRef]

10. Li, J.; Zakharov, Y.V.; Henson, B. Multibranch autocorrelation method for Doppler estimation in underwater acoustic channels. IEEE J. Ocean. Eng. 2018, 3, 1099-1113. [CrossRef]

11. Li, J.; Zakharov, Y.V. Efficient use of space-time clustering for underwater acoustic communications. IEEE J. Ocean. Eng. 2018, 43, 173-183. [CrossRef]

12. Stojanovic, M. Design and capacity analysis of cellular-type underwater acoustic networks. IEEE J. Ocean. Eng. 2008, 33, 171-181. [CrossRef]

13. Srinivasan, B.; Rodoplu, V. Capacity of underwater acoustic OFDM cellular networks. In Proceedings of the IEEE OCEANS, Sydney, Australia, 24-27 May 2010.

14. Yang, T.C. Code division multiple access based multiuser underwater acoustic cellular network. J. Acoust. Soc. Am. 2011, 130, 2347. [CrossRef]

15. Akhoundi, F.; Salehi, J.A.; Tashakori, A. Cellular underwater wireless optical CDMA network: Performance analysis and implementation concepts. IEEE Trans. Commun. 2015, 63, 882-891. [CrossRef]

16. Akhoundi, F.; Mohammad, V.J.; Navid, B.H.; Hamzeh, B.; Amir, M.; Jawad, A.S. Cellular underwater wireless optical CDMA network: Potentials and challenges. IEEE Access 2016, 4, 4254-4268. [CrossRef]

17. Burdic, W.S. Underwater Acoustic System Analysis; Peninsula Publishing: Newport Beach, CA, USA, 2002.

18. Tan, H.P.; Gabor, A.F.; Eu, Z.A.; Seah, W.K.G. A wide coverage positioning system (WPS) for underwater localization. In Proceedings of the 2010 IEEE International Conference on Communications, Cape Town, South Africa, 23-27 May 2010.

19. Girod, L.; Bychkovskiy, V.; Elson, J.; Estrin, D. Locating tiny sensors in time and space: A case study. In Proceedings of the IEEE International Conference on Computer Design: VLSI in Computers and Processors, Freiburg, Germany, 18 September 2002.

20. Harter, A.; Hopper, A.; Steggles, P.; Ward, A.; Webster, P. The anatomy of a context-aware application. Wirel. Netw. 2002, 8, 187-197. [CrossRef]

21. Girod, L.; Estrin, D. Robust range estimation using acoustic and multimodal sensing. In Proceedings of the IEEE/RSJ International Conference on Intelligent Robots and Systems, Expanding the Societal Role of Robotics in the the Next Millennium, Maui, HI, USA, 29 October-3 November 2001.

22. Niculescu, D.; Nath, B. Ad hoc positioning system (APS) using AOA. In Proceedings of the Twenty-second Annual Joint Conference of the IEEE Computer and Communications Societies, San Francisco, CA, USA, 30 March-3 April 2003.

23. Chandrasekhar, V.; Seah, W.K.G. Area localization scheme for underwater sensor networks. In Proceedings of the OCEANS 2006-Asia Pacific, Singapore, 16-19 May 2006.

24. Zhou, Y.; He, J.; Chen, K.; Chen, J.; Liang, A. An area localization Scheme for large scale Underwater Wireless Sensor Networks. In Proceedings of the 2009 WRI International Conference on Communications and Mobile Computing, Kunming, China, 6-8 January 2009.

25. Chandrasekhar, V.; Seah, W.K.G.; Choo, Y.S.; Ee, H.V. Localization in underwater sensor networks-Survey and challenges. In Proceedings of the 1st ACM International Workshop on Underwater Networks, Atlanta, GA, USA, 23-25 October 2019.

26. Zhou, Z.; Cui, J.H.; Zhou, S. Efficient localization for large-scale underwater sensor networks. Ad. Hoc. Netw. 2010, 8, 267-279. [CrossRef]

27. Mirza, D.; Schurgers, C. Motion-aware self-localization for underwater networks. In Proceedings of the Third ACM International Workshop on Underwater Networks, San Francisco, CA, USA, 15 September 2008. 
28. Diamant, R.; Lampe, L. Underwater localization with time-synchronization and propagation speed uncertainties. IEEE Trans. Mob. Comput. 2013, 12, 1257-1269. [CrossRef]

29. Erol-Kantarci, M.; Mouftah, H.T.; Oktug, S. Localization techniques for underwater acoustic sensor networks. IEEE Commun. Mag. 2010, 48, 152-158. [CrossRef]

30. Zhang, B.; Yu, F.; Zhang, Z. An improved localization algorithm for wireless sensor network using a mobile anchor node. In Proceedings of the 2009 Asia-Pacific Conference on Information Processing, Shenzhen, China, 18-19 July 2009.

31. Yun, S.; Lee, J.; Chung, W.; Kim, E.; Kim, S. A soft computing approach to localization in wireless sensor networks. Expert Syst. Appl. 2009, 36, 7552-7561. [CrossRef]

32. Gogolak, L.; Pletl, S.; Kukolj, D. Indoor fingerprint localization in WSN environment based on neural network. In Proceedings of the 2011 IEEE 9th International Symposium on Intelligent Systems and Informatics, Subotica, Serbia, 8-10 September 2011.

33. Rahman, M.S.; Park, Y.; Kim, K.D. RSS-based indoor localization algorithm for wireless sensor network using generalized regression neural network. Arab. J. Sci. Eng. 2012, 37, 1043-1053. [CrossRef]

34. Azenha, A.; Peneda, L.; Carvalho, A. A neural network approach for Radio Frequency based indoors localization. In Proceedings of the IECON 2012-38th Annual Conference on IEEE Industrial Electronics Society, Montreal, QC, Canada, 25-28 October 2012.

35. Luoh, L. ZigBee-based intelligent indoor positioning system soft computing. Soft Comput. 2014, 18, 443-456. [CrossRef]

36. Gogolak, L.; Pletl, S.; Kukolj, D. Neural network-based indoor localization in WSN environments. Acta Polytech. Hung. 2013, 10, 221-235.

37. Payal, A.; Rai, C.S.; Reddy, B.V.R. Artificial neural networks for developing localization framework in wireless sensor networks. In Proceedings of the 2014 International Conference on Data Mining and Intelligent Computing (ICDMIC), Delhi, India, 5-6 September 2014.

38. Chuang, P.J.; Jiang, Y.J. Effective neural network-based node localisation scheme for wireless sensor networks. IET Wirel. Sens. Syst. 2014, 4, 97-103. [CrossRef]

39. Zepernick, H.J.; Finger, A. Pseudo Random Signal Processing: Theory and Application; Wiley: Chichester, UK, 2005.

40. Richards, M.A. Fundamentals of Radar Signal Processing; McGraw-Hill: New York, NY, USA, 2015.

41. Sesia, S.; Toufik, I.; Baker, M. LTE-The UMTS Long Term Evolution: From Theory to Practice; Wiley: Chichester, UK, 2011.

42. Evolved Universal Terrestrial Radio Access (E-UTRA). Physical Channels and Modulation (3GPP TS 36.211 version 12.3.0 Release 12). Available online: https://www.etsi.org/deliver/etsi_ts/136200_136299/136211/12.03.00_60/ts_136211v120300p.pdf (accessed on 30 July 2021).

43. Mason, S.F.; Berger, C.R.; Zhou, S.; Willett, P. Detection, synchronization, and Doppler scale estimation with multicarrier waveforms in underwater acoustic communication. IEEE J. Sel. Areas Commun. 2008, 26, 1638-1649. [CrossRef]

44. Schmidl, T.M.; Cox, D.C. Robust frequency and timing synchronization for OFDM. IEEE Trans. Signal Process. 1997, 45, 1613-1621. [CrossRef]

45. Li, X.; Ng, F.; Han, T. Carrier frequency offset mitigation in asynchronous cooperative OFDM transmissions. IEEE Trans. Signal Process. 2008, 56, 675-685. [CrossRef]

46. Zhou, S.; Wang, Z. OFDM for Underwater Acoustic Communications; Wiley: Chichester, UK, 2014.

47. Asim, M.; Khan, M.S.; Im, T.H.; Cho, Y.S. Cell ID and timing estimation techniques for underwater acoustic cellular systems in High-Doppler environments. Sensors 2020, 20, 4147. [CrossRef]

48. Cho, Y.S.; Kim, J.; Yang, W.Y.; Kang, C.G. MIMO-OFDM Wireless Communications with MATLAB; Wiley: Chichester, UK, 2011.

49. Urick, R.J. Principles of Underwater Sound; McGraw-Hill: New York, NY, USA, 1983.

50. Catipovic, J.; Baggeroer, A.; Heydt, K.V.D.; Koelsch, D. Design and performance analysis of a digital acoustic telemetry system for the short range underwater channel. IEEE J. Ocean. Eng. 1984, 9, 242-252. [CrossRef]

51. Wynn, R.B.; Bett, B.J.; Evans, A.J.; Griffiths, G.; Huvenne, V.A.I.; Jones, A.R.; Palmer, M.R.; Dove, D.; Howe, J.A.; Boyd, T.J. Investigating the Feasibility of Utilizing AUV and Glider Technology for Mapping and Monitoring of the UK MPA Network; Final report for Defra project MB0118; National Oceanography Centre (NOC): Southampton/Liverpool, UK, 2012. 